\title{
SOBRE LA TAXONOMÍA Y BIOLOGÍA DE CYCLOCEPHALA MANNHEIMSI ENDRÖDI, 1964 (COLEOPTERA: SCARABAEIDAE: DYNASTINAE), NUEVO REGISTRO PARA COLOMBIA
}

\author{
HÉCTOR JAIME GASCA-ÁLVAREZ \\ Corporación Sentido Natural. Calle 124 \# 70H - 79. Bogotá Colombia \\ Programa de Doctorado en Ciencias. Línea de Sistemática y Biodiversidad. Red de Interacciones \\ Multitróficas. Instituto de Ecología, A.C. Carretera antigua a Coatepec 351, El Haya, 91070, Xalapa, \\ Veracruz, México \\ $<$ hjgasca@sentidonatural.org $><$ hector.gasca@posgrado.inecol.edu.mx $>$
}

Gasca-Álvarez, H. J. 2014. Sobre la taxonomía y biología de Cyclocephala mannheimsi Endrödi, 1964 (Coleoptera: Scarabaeidae: Dynastinae), nuevo registro para Colombia. Acta Zoológica Mexicana (n.s.), 30(1): 174-187.

RESUMEN. Se reporta por primera vez para Colombia a Cyclocephala mannheimsi Endrödi, 1964, especie conocida hasta el momento para Bolivia, Ecuador y Perú. Se realiza la re-descripción de la especie a partir de la revisión de 103 ejemplares. Se describen e ilustran los patrones de pigmentación observados analizando las formas predominantes y discutiendo las posibles causas que influyen en la variación de coloración de esta especie y de otras especies de Cyclocephala.

Palabras clave: Patrones de pigmentación, variación intraespecifica, Polifenismo.

Gasca-Álvarez, H. J. 2014. On the taxonomy and biology of Cyclocephala mannheimsi Endrödi, 1964 (Coleoptera: Scarabaeidae: Dynastinae), new record for Colombia. Acta Zoológica Mexicana (n.s.), 30(1): 174-187.

ABSTRACT. Cyclocephala mannheimsi Endrödi, 1964 is recorded for the first time in Colombia, currently known from Bolivia, Ecuador and Peru. Species redescription is provided based in 103 exemplars. Color patterns observed are described and illustrated analyzing dominant forms and discuss possible causes that influence the variation of pigmentation in that species and other Cyclocephala species.

Key word: Pigmentation patterns, intraspecific variation, Polyphenism.

\section{INTRODUCCIÓN}

La especie Cyclocephala mannheimsi fue descrita por Endrödi a partir de 3 ejemplares provenientes del Ecuador sin localidad definida. En la descripción original el autor menciona caracteres básicos para la identificación de esta especie como la co-

Recibido: 09/07/2013; aceptado: 25/09/2013. 
loración del cuerpo, forma del clípeo, presencia de sutura frontal, forma del pronoto, presencia de 3 manchas longitudinales en cada élitro, e ilustra la región frontal de los parámeros del macho (Endrödi 1964). Más adelante en su obra The Dynastinae of the World, Endrödi añade otros caracteres en la descripción, y menciona la presencia de 2 manchas alargadas en el pronoto, señalando nuevamente la presencia de 3 manchas en cada élitro e ilustra el patrón de coloración básico. De igual manera amplía la distribución de la especie para Bolivia sin mencionar una localidad específica (Endrödi 1985).

La variación en el patrón de pigmentación ha sido observada en varias especies de Cyclocephala (Endrödi 1966, 1985; Morón 1977 a, b; Ratcliffe \& Delgado 1990, García-Luna et al. 2002), lo que ha ayudado a su identificación, sobre todo en individuos de la misma especie que presentan variaciones notables en su patrón de coloración (Moore 2011). Aún se desconoce cuál es la causa de esta variación, sin embargo, algunos autores sugieren que este fenómeno puede deberse a factores como la temperatura y humedad del suelo, que actúan durante el proceso de endurecimiento y pigmentación de la cutícula en la fase de transición de pupa a adulto, incrementando o disminuyendo la superficie melanizada en élitros y pronoto (García-Luna et al. 2002).

El género Cyclocephala es uno de los más diversos en Colombia dentro de la subfamilia Dynastinae. Restrepo et al. (2003) reportaron 67 especies para el país, Young \& Le Tirant describieron una nueva especie proveniente del departamento del Huila, y Ratcliffe (2008) describió una nueva especie a partir de un único ejemplar colectado en la amazonia Colombiana. Conocida para Ecuador, Bolivia y Perú, $C$. mannheimsi es registrada por primera vez para Colombia en el presente trabajo. A partir del estudio de material depositado en colecciones de referencia de entomología, y de material colectado en sectores aledaños a la ciudad de Leticia, Amazonas, se realiza la re-descripción de la especie, analizando las variaciones en el patrón de pigmentación de machos y hembras y aportando información sobre su biología e historia natural.

\section{MATERIAL Y MÉTODOS}

Los ejemplares examinados para el presente trabajo, procedieron de las siguientes colecciones:

CHJG: Colección Personal Héctor Jaime Gasca Álvarez, Bogotá D.C.

ICN: Museo de Historia Natural, Instituto de Ciencias Naturales, Universidad Nacional de Colombia, Bogotá D.C. (Germán Amat-García)

IAvH: Colecciones Biológicas, Instituto de Investigación de Recursos Biológicos Alexander Von Humboldt, Villa de Leyva-Boyacá (Claudia Medina)

UNAB: Museo Entomológico Facultad de Agronomía, Universidad Nacional de Colombia, Bogotá D.C. (Francisco Serna y Erika Vergara) 
Adicionalmente se examinaron ejemplares obtenidos en jornadas de colecta realizadas en áreas de bosque intervenido y cultivos indígenas (chagras), localizadas en la comunidad Uitoto del $\mathrm{Km} \mathrm{11}$, sobre la vía que conduce de la ciudad de Leticia al municipio de Tarapacá, departamento del Amazonas. Las colectas se realizaron entre los meses de agosto y octubre de 2001, empleando trampa de luz blanca de tipo pantalla cuyo tiempo de acción fue desde las 6:00 pm hasta las 12:00 am.

Para el análisis del patrón de pigmentación en machos y hembras de C. mannheimsi se separaron todos los individuos con variaciones de pigmentación tanto en el pronoto como en los élitros, para formar una muestra de referencia con todas las variedades posibles. Se formaron subgrupos o patrones de coloración de acuerdo con la forma y el número de manchas; a cada uno de los patrones observados se le asignó un número romano.

\section{RESULTADOS Y DISCUSIÓN}

\section{Cyclocephala mannheimsi Endrödi, 1964}

Cyclocephala mannheimsi Endrödi, 1964: 444

(Fig. 1-8)

Re-descripción: Largo 13.34-15.72 mm; ancho 6.20-868 mm. Color: Cuerpo amarillento. Frente rojizo oscuro. Pronoto usualmente con dos manchas longitudinales, paralelas, marrón oscuro, con dos manchas circulares situadas cerca del borde lateral. Élitros usualmente con tres manchas alargadas de color marrón oscuro presentando variación en el patrón de pigmentación. Pigidio marrón oscuro, región central amarillenta en los machos (Fig. 2), oscura en las hembras (Fig. 3). Fémures de color amarillento, tibias con coloración más oscura.

Machos: Cabeza: Frente con puntuaciones densas. Clípeo parabólico, superficie fuertemente punteada, ápice truncado, con margen basal. Sutura frontoclipeal bien definida, con dos manchas circulares de color marrón (Fig. 4). Canto ocular con ápice redondeado, superficie con 4 a 5 setas cortas. Antena con 10 artejos, lamelas subiguales en tamaño. Mandíbulas moderadamente anchas, ápice fuertemente arqueado, redondeado, borde interno con proyección triangular aplanada. Pronoto: Superficie finamente punteada. Puntuaciones moderadas, de tamaño pequeño, dispuestas irregularmente. Borde anterior con margen basal. Élitros: Superficie con 6 a 7 hileras de puntuaciones en cada élitro. Puntuaciones profundas, densas, de tamaño variable. Con pequeñas setas en los tercios laterales. Disco con una pequeña protuberancia. Húmero con pequeñas sedas cortas. Pigidio: Superficie rugosa, punteada, Puntuaciones pequeñas. Ápice setígero, setas densas y largas. Superficie convexa en vista lateral. Región ventral: Proceso prosternal largo, triangular, ápice redondeado, setígero, 


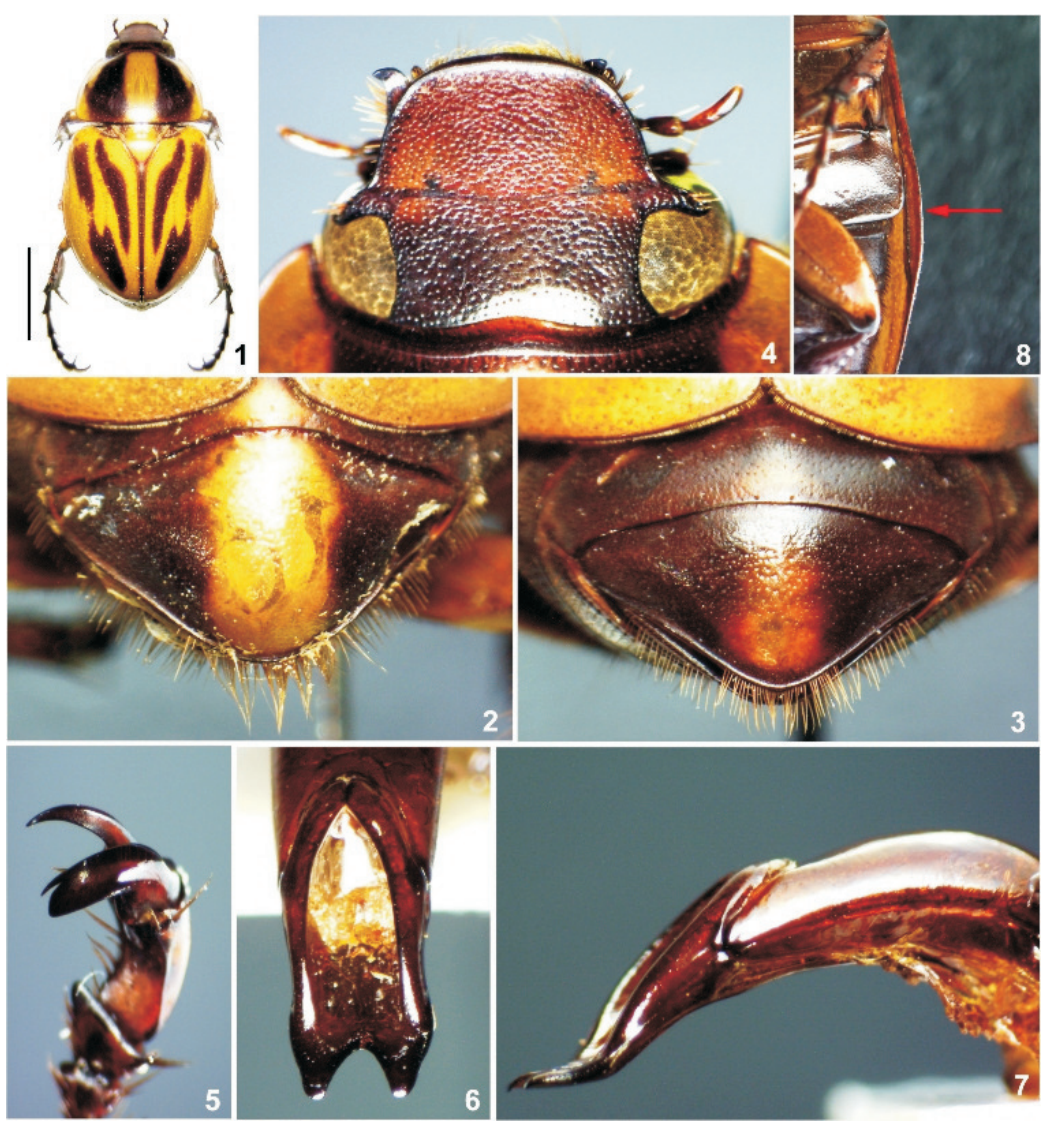

Figuras 1-8. Cyclocephala mannheimsi: 1) Hábito. 2) Pigidio macho. 3) Pigidio hembra. 4) Clípeo. 5) Uña anterior macho. 6) Parámeros vista frontal. 7) Parámeros vista lateral. 8) Epipleura hembra vista ventral. Línea de escala: $5 \mathrm{~mm}$.

sedas densas y largas. Ápice con un pequeño tubérculo redondo en vista dorsal. Patas: Tibia anterior bidentada. Tarsos anteriores engrosados. Tarsómero 4 ensanchado, tarsómero 5 engrosado, curvado. Uña mayor robusta, fuertemente arqueada, con hendidura apical pronunciada; la rama mayor redondeada en el ápice (Fig. 5). Parámeros: alargados, curvos hacia el ápice, ápices moderadamente redondeados (Fig. 6 y 7).

Hembras: Como en los machos excepto en: Cabeza: setas de la superficie del canto ocular ausentes. Élitros: sedas ausentes, puntuación moderada. Pigidio: En vista lateral, superficie ligeramente convexa a plana. Piernas: Tibia anterior tridentada. Tarsos anteriores simples. Uñas simples. Epipleura: Ligeramente ensanchada, estrecha desde los esternitos abdominales (Fig. 8). 
Distribución: Ecuador, Bolivia y Perú. Los ejemplares examinados constituyen un NUEVO REGISTRO para el país. Distribución en Colombia: Hasta el momento $C$. mannheimsi se conoce para los departamentos de Amazonas y Nariño.

Diagnosis. Debido al patrón de pigmentación en pronoto y élitros, sobre todo en las hembras, C. mannheimsi puede asemejarse a especies como C. amazona (Linnaeus, 1767), C. multiplex Casey, 1915, C. pardolocarnoi Dechambre, 1995, C. prolongata Arrow, 1902 y $C$. stictica Burmeister, 1847. La forma del clípeo, el examen de la genitalia masculina y el patrón de coloración característico en los machos (patrón VII), son caracteres que permiten la identificación de C. mannheimsi. En las hembras, algunos caracteres pueden ser utilizados para diferenciar especies afines a C. mannheimsi (Cuadro 1).

Biología. Los adultos generalmente son atraídos a la luz blanca. Durante colectas realizadas en áreas de chagras (cultivos indígenas) en la amazonia colombiana fueron capturados gran cantidad de individuos, empleando trampa de luz tipo pantalla durante las 6:30 pm y las 10:30 pm. Aunque sus estados larvales se desconocen, es posible que las larvas estén asociadas a yuca Manihot sculenta Crantz (Euphorbiaceae) y piña Ananas comosus (L.) (Bromeliaceae), cultivos presentes en las chagras, posiblemente compitiendo por recursos alimenticios en las mismas áreas con Cyclocephala amazona (Gasca 2005).

Material examinado: 41 especímenes revisados de colecciones $(8 \hat{\circ}$ y 33 \% $)$. Amazonas: Araracuara, 21.ago.1977, Col: Restrepo, R., 1ð, 4우 [028942, 036526, 036528, 036643, 036644, ICN]; Macarena, Los Micos, 15.oct.1972, Col: Rojas, J., 1 ㅇ [028943, ICN]; Leticia, Km 2 vía Tarapacá, IMANI, UN, 7.ago.2001, 80 m,

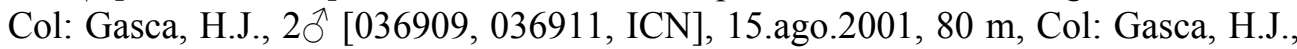
2 ㅇ [036910, 036912, ICN], 27.oct.2002, 80 m, Col: Gasca, H.J., 1 ㅇ [306, CHJG]; Km 11 vía Tarapacá, Sendero Rio Tacana, 16.ago.2001, 80 m, Col: Gasca, H.J., 1 + [036908], 22.ago.2001, Col: Gasca, H.J., 2 ô 3 + [036906, 036904, 036905, 036907, ICN; 291, CHJG], 23.ago.2001, Col: Gasca, H.J., 4ㅇ [036900, 036901, 036902, 036903, ICN], 24.ago.2001, 120 m, Col: Gasca, H.J., 5 ㅇ [036895, 036896, 036897, 036898 036899, ICN], 25.ago.2001, 100 m, Col: Gasca, H.J., $1 \overbrace{}^{\curvearrowright}$ [292, CHJG]. Nariño: Territorio Kofán, $00^{\circ} 30^{\prime} 07^{\prime}$ 'N, $77^{\circ} 13^{\prime} 43^{\prime}$ 'W, sep.1998, 700 m, Col: González. E., $1 \hat{\jmath}, 1+$ [UNAB], $1 \hat{\jmath}, 6+$ [IAvH].

Comentarios: García-Luna et al., (2002) mencionan el nombre de C. mannerheimi Endrödi, el cual pertenece a un grupo de especies de Cyclocephala que se caracterizan por presentar la base del pronoto marginada, protibias tridentadas, élitros glabros, maza antenal masculina más corta que la suma de los artejos precedentes, pronoto y élitros con manchas o franjas oscuras y uñas protarsales internas de los machos con el ápice hendido. Sin embargo, debe ser entendido que la especie a la cual se hace referencia es Cyclocephala mannheimsi Endrödi, ya que el nombre "mannerheimi" corresponde a la especie Agaocephala mannerheimi Castaleneu, 1932, perteneciente 


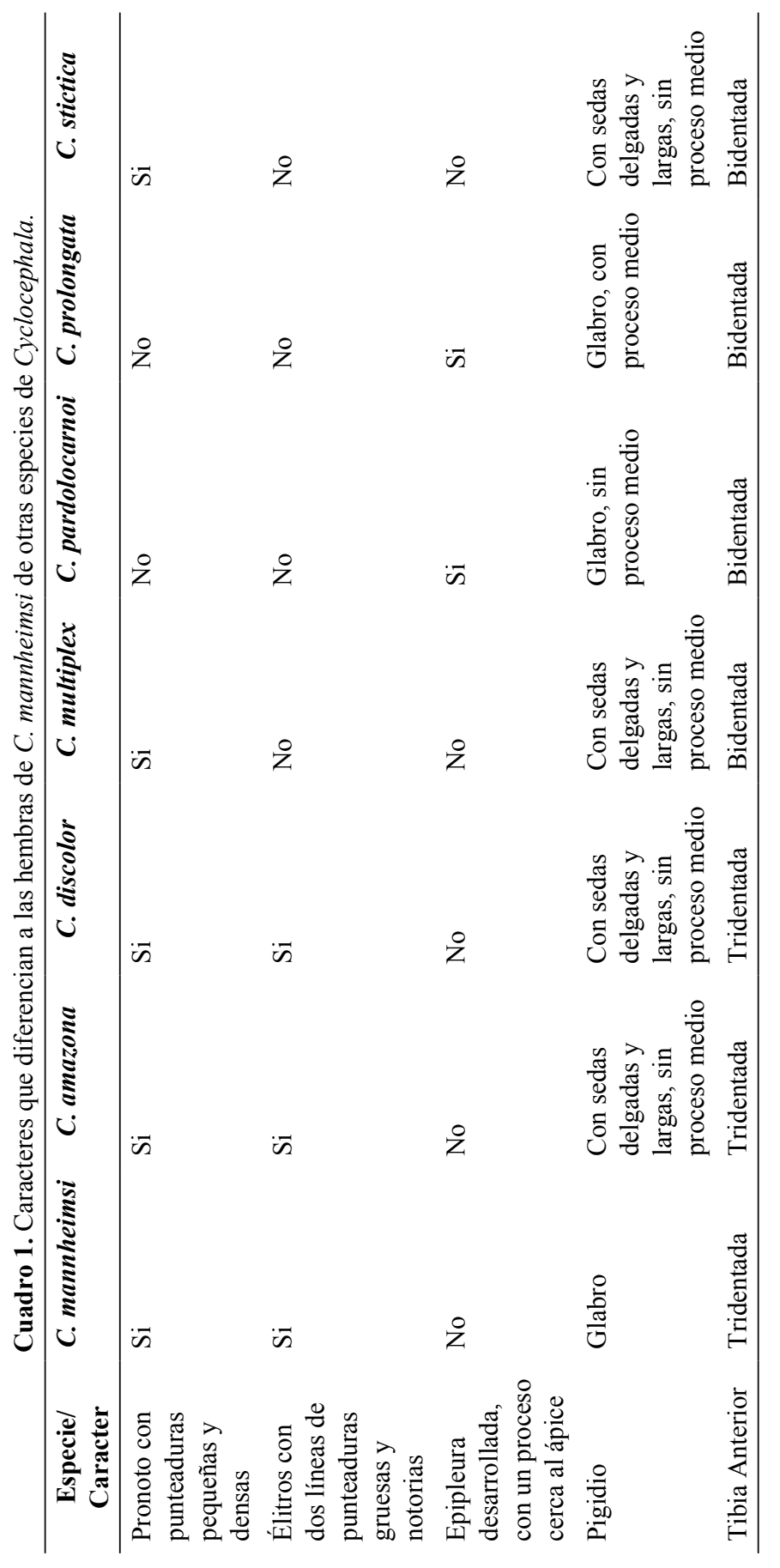


a la tribu Agaocephalini. Los caracteres diagnósticos del grupo mencionados se ajustan a C. mannheimsi.

Variación del patrón de pigmentación: Se revisaron un total de 103 ejemplares (14 machos y 89 hembras), de los cuales 62 fueron colectados con trampa de luz tipo pantalla en la comunidad del Km 11 (vía Leticia-Tarapacá) Amazonas. El análisis del material examinado mostró siete variaciones definidas en el patrón de pigmentación del pronoto y de los élitros (Fig. 9). En todas las variaciones la cabeza es de color oscuro y la superficie del clípeo es de color amarillento difuminado.

Patrón I: Pronoto con dos manchas gruesas longitudinales y paralelas de color marrón oscuro. Con dos manchas circulares cerca del borde lateral. Élitros con una pequeña mancha longitudinal y delgada (Fig. 9A). Esta variación representa el 2.91\% del material examinado.

Patrón II: Pronoto con patrón de pigmentación como en la variación I. Élitros con dos manchas gruesas diagonales y curvas extendiéndose hasta la mitad de los élitros
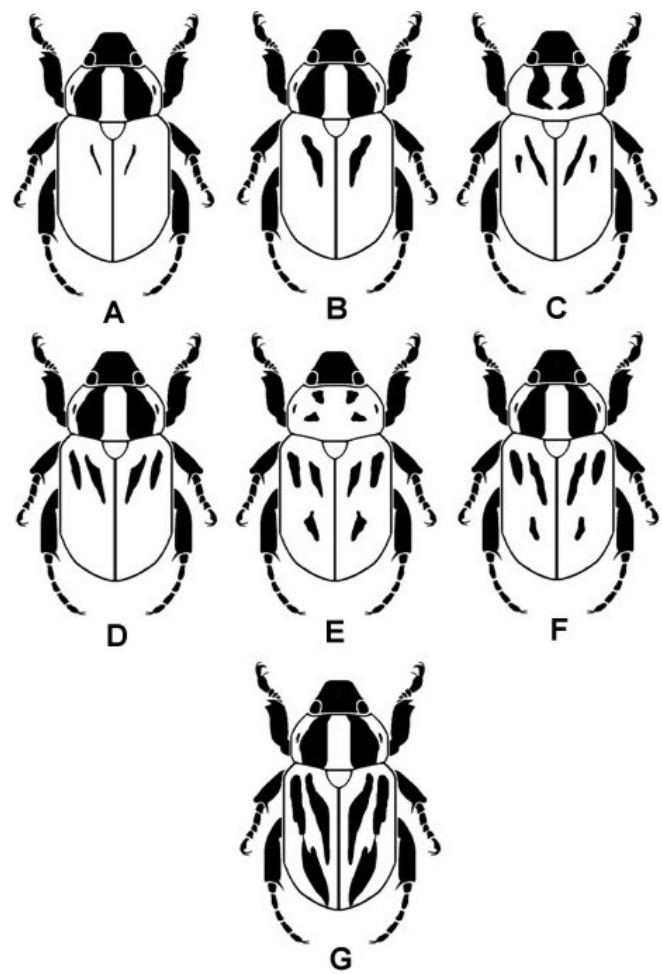

Figura 9. Variación en el patrón de pigmentación de Cyclocephala mannheimsi.

A) Patrón I. B) Patrón II. C) Patrón III. D) Patrón IV.

E) Patrón V. F) Patrón VI. G) Patrón VII. 
(Fig. 9B). Esta variación representa el 11.65\% del material examinado.

Patrón III: Pronoto con dos manchas gruesas y paralelas terminando en una muesca, sin extenderse hasta el borde posterior. Manchas circulares laterales ausentes. Élitros con cuatros manchas, dos manchas rectas y diagonales y dos manchas pequeñas de forma irregular (Fig. 9C). Esta variación corresponde al 4.85\% del material examinado.

Patrón IV: Pronoto con patrón de pigmentación como en la variación I y II. Cada élitro con dos manchas gruesas y de forma irregular localizadas en el primer tercio de la superficie elitral (Fig. 9D). Esta variación corresponde al 34.95\% del material examinado.

Patrón V: Pronoto con seis manchas, dos manchas circulares hacia los bordes laterales y cuatro manchas de forma irregular localizadas en la región central. Élitros con seis manchas gruesas de forma irregular con tamaño variable (Fig. 9E). Esta variación corresponde al $3.88 \%$ del material examinado.

Patrón VI: Pronoto con patrón de pigmentación como en la variación I, II y IV. Élitros con seis manchas, dos manchas de forma generalmente elíptica localizadas en el disco, y cuatro manchas más o menos alargadas y de forma irregular (Fig. 9F). Esta variación corresponde al $28.16 \%$ del material examinado.

Patrón VII: Pronoto con patrón de pigmentación como en la variación I, II, IV y VI. Élitros con seis manchas gruesas, muy alargadas, curvas y de forma irregular, extendiéndose hasta el ápice. En la mayoría de los ejemplares se observó que las dos manchas laterales de cada élitro se encontraban conectadas por una fina línea oscura (Fig. 9G). Esta variación corresponde al 28.16\% del material examinado.

Dentro de la muestra analizada, la variación de coloración dominante correspondió al patrón IV, mientras que las variaciones con poca pigmentación en el pronoto y los élitros fueron las menos frecuentes (Fig. 10). Todos los machos examinados en la muestra presentaron el patrón de coloración VII, el cual tiene el mayor grado de pigmentación oscura en los élitros. Esto podría sugerir la existencia de otro carácter de dimorfismo sexual en C. mannheimsi, aparte del engrosamiento de los protarsos en los machos, el ensanchamiento de la epipleura en las hembras y la diferencia de coloración en el pigidio.

La variación de la coloración intraespecífica es un carácter morfológico común en muchas especies del género Cyclocephala. Los diferentes patrones de pigmentación, en individuos de una misma especie, pueden presentarse tanto en el pronoto como en los élitros, y en algunas ocasiones en la superficie del pigidio. Estos patrones se manifiestan generalmente con la presencia de manchas de color oscuro, circulares o de forma irregular, o en otros casos, con bandas melanizadas gruesas o delgadas. Los patrones de manchas en pronoto y élitros de varias especies de Cyclocephala fueron ilustradas por Endrödi (1985), con el fin de facilitar la identificación de especies o grupos de especies. Sin embargo, una misma especie puede presentar más de 20 va- 


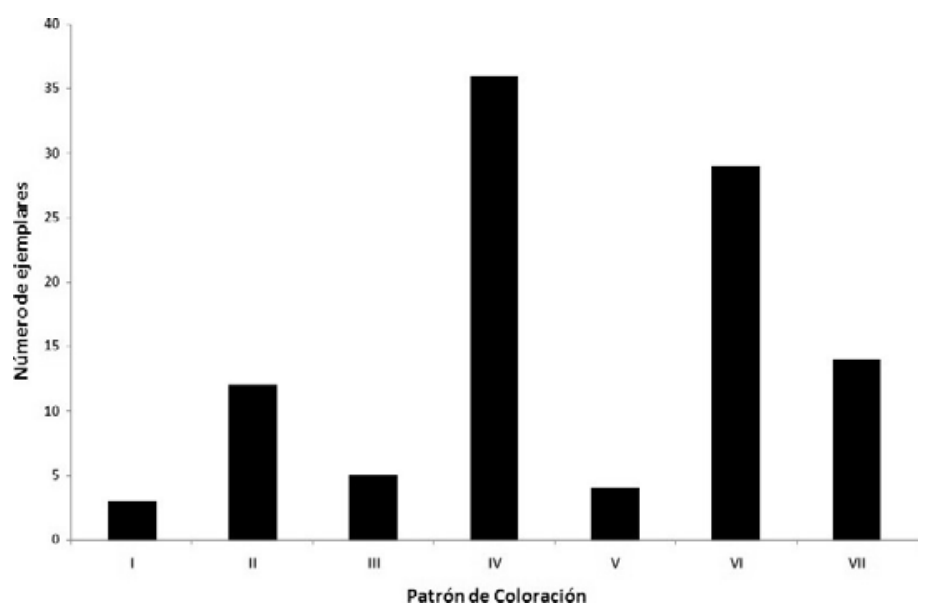

Figura 10. Relación de los patrones de pigmentación de Cyclocephala mannheimsi en la muestra estudiada.

riaciones, las cuales se pueden definir por la presencia o ausencia de manchas, forma, cantidad y posición en los élitros (Cuadro 2). De igual manera, pueden presentarse formas oscuras, que algunos autores como Endrödi las ha categorizado como "aberraciones".

Estos patrones de pigmentación generalmente se han usado para diferenciar muchas especies de Cyclocephala, sin embargo autores como Casey (1915) aplicaron en su concepto de especie las diferencias de coloración, describiendo diferentes especies que posteriormente fueron sinonimizadas con especies como Cyclocephala sexpunctata Laporte, 1840 o Cyclocephala mafaffa Burmeister, 1847. De aquí la importancia de analizar una serie representativa de ejemplares de una especie en donde se detecte algún tipo de variación en su patrón de coloración, con el fin de evaluar si se trata de una misma especie o de grupos de especies. Sin embargo, en aquellas especies con muchas variaciones en su patrón de pigmentación como C. maffafa o C. sexpunctata (Cuadro 2), puede presentarse también variaciones en la forma de los parámeros de los machos (Ratcliffe 2003, Moore 2011). Esto ha generado que se realicen estudios de filogenia y análisis molecular empleando diferentes fenotipos, los cuales han demostrado la existencia de polifenismo en especies del género Cyclocephala (Moore 2011).

A pesar de que las diferentes formas de coloración son evidentes en la naturaleza, aún se desconocen los verdaderos mecanismos que causan la variación intraespecífica en la melanización de la cutícula de los insectos. La pigmentación es uno de los ejemplos más simples y comunes de la biodiversidad en la naturaleza (True 2003), en donde se pueden presentar tanto polimorfismos intraespecíficos como diferencias 


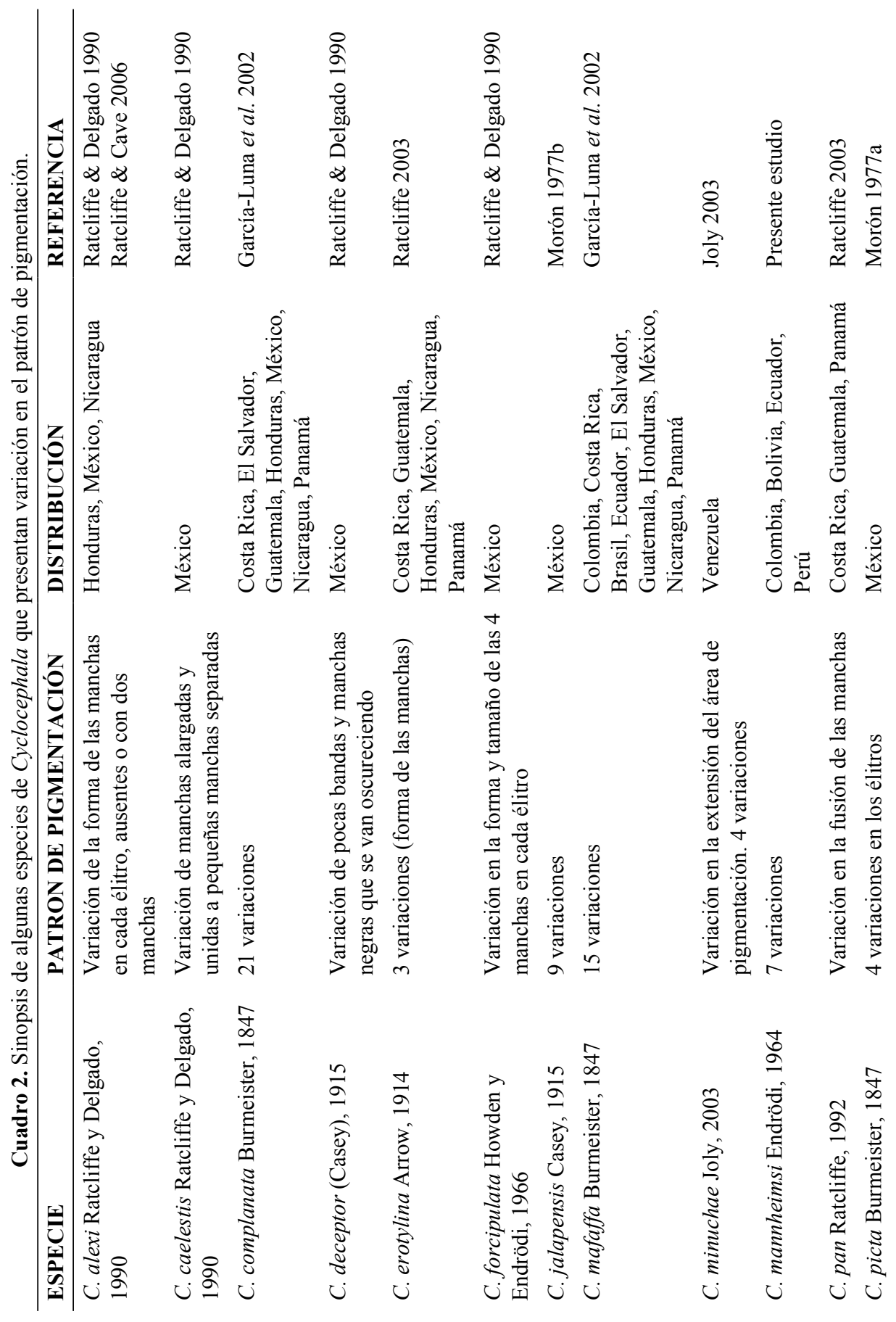




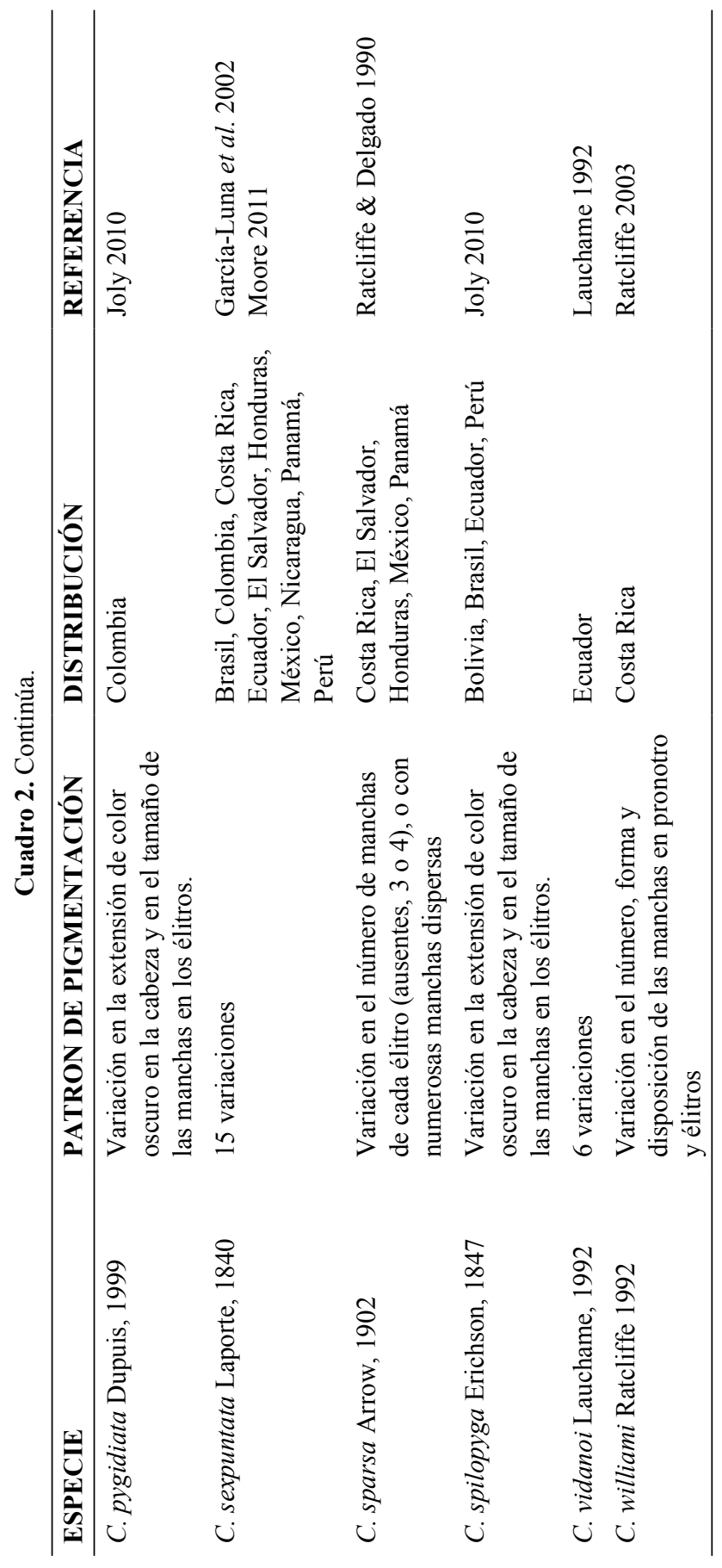


fijas entre especies estrechamente relacionadas, lo que puede influir en muchos aspectos de la biología de los insectos. Desde el punto de vista ecológico, se ha sugerido que la pigmentación desempeña un papel importante en la termorregulación, la resistencia a la desecación, la radiación ultravioleta, parasitismo, aposematismo y en comportamientos de cortejo (Wittkopp et al. 2003, True 2003). La melanización de la cutícula en insectos está asociada al proceso de esclerotización durante la ecdisis y el desarrollo pupal en la holometabolia, donde diferentes juegos de enzimas con sus respectivos catalizadores, actúan a través de patrones determinados genéticamente (Hopkins \& Kramer 1992).

Para el caso de la pigmentación en Cyclocephala, Garcia-Luna et al. (2002) sugirieron, analizando la amplitud y la frecuencia de la variación de la pigmentación en tres especies del género, que el incremento y diferencias de coloración en élitros y pronoto, podría estar relacionado con cambios en la temperatura y humedad del suelo durante los procesos finales del estado de pupa y esclerotización de la cutícula en los adultos. Esta hipótesis está basada en las localidades de captura de las muestras analizadas, y radica en que existe un predominio de determinado patrón de pigmentación (formas más obscuras) en zonas frías, mientras que en las zonas cálidas predominan otros patrones de pigmentación (formas menos pigmentadas), dando lugar a una relación de temperatura, altitud y humedad sobre la intensificación del melanismo. Sin embargo, para el caso de C. mannheimsi, de acuerdo con su distribución conocida, ha sido colectada únicamente en zonas cálidas que no superan los $1000 \mathrm{~m}$ de elevación, y en donde tanto las formas menos pigmentadas (patrón I y V) como las formas más pigmentadas (patrón VI y VII) pueden ser encontradas en el mismo lugar de colecta (Cuadro 3), por lo que las condiciones de temperatura y humedad del suelo, no estarían influyendo en el aumento o disminución de la melanización de la cutícula. De esta manera, puede sugerirse que otros factores como la calidad y cantidad del alimento que consumen las larvas durante el ciclo de vida, o procesos de genes dominantes o recesivos que expresen el fenotipo para la condición de pigmentación, pudieran estar actuando en la formación de especies con polifenismo en el género Cyclocephala, y posiblemente en otros géneros de Cyclocephalini.

Cuadro 3. Relación del patrón de coloración y lugar de colecta de Cyclocephala mannheimsi.

\begin{tabular}{|c|c|c|}
\hline Lugar de colecta & Patrón de pigmentación & Sexos \\
\hline Araracuara & VI -VII & $1 \jmath^{\lambda}$ ㅇ \\
\hline Macarena & I & 1 우 \\
\hline Leticia Km 2 & $\mathrm{VI}-\mathrm{VII}$ & $2 \lesssim 30$ \\
\hline Leticia Km 11 & I - II - IV - V- VI - VII & $9 \overbrace{}^{\lambda} 75 q$ \\
\hline Territorio Kofán & II - III - VI - VII & $2 \widehat{\jmath} 7$ 우 \\
\hline
\end{tabular}


AGRADECIMIENTOS. A Walter Morales, habitante de la comunidad Uitoto del Km 11, por su importante labor como auxiliar de campo en las jornadas de colecta. A los curadores de las colecciones visitadas por permitir la revisión del material. Agradecimientos especiales a dos evaluadores anónimos por sus valiosos comentarios y sugerencias al manuscrito. Este trabajo hace parte del programa de investigación de la Corporación Sentido Natural.

\section{LITERATURA CITADA}

Casey, T. L. 1915. Memoirs on the Coleoptera, VI. Lancaster, Pa. pp. 109-137.

Endrödi, S. 1964. Eine Reihe von neuen Cyclocephala-Arten. Folia Entomologica Hungarica (N.S.), 17: 433-470.

Endrödi, S. 1966. Monographie der Dynastinae (Col. Lam.) I. Teil. Entomologische Abhandlungen Museum Tierkunde, Dresden, Bd., 33: 1-457.

Endrödi, S. 1985. The Dynastinae of the World. Dr W. Junk; Dordrecht, Netherlands, 800 p.

García-Luna, D., Morón, M. A. \& Rojas-Gómez, C. V. 2002. Variación en los patrones de pigmentación en tres especies de Cyclocephala Burmeister (Coleoptera: Melolonthidae: Dynastinae). Folia Entomológica Mexicana, 41: 129-148.

Gasca, H. J. 2005. El significado de los escarabajos (Coleoptera: Scarabaeoidea) en una comunidad Uitoto de Leticia, Amazonas (Colombia): Una exploración preliminar a su conocimiento etnoentomológico. Boletín Sociedad Entomológica Aragonesa, 36: 309-315.

Hopkins, T. L. \& Kramer, K. J. 1992. Insect cuticule sclerotization. Annual Review of Entomolgy, 37 : 273-302.

Joly, L. J. 2003. Cyclocephala minuchae, nueva especie de Venezuela y redescripción de C. vincentiae Arrow (Coleoptera: Scarabaeidae: Dynastinae: Cyclocephalini). Entomotrópica, 18: 37-47.

Joly, L. J. 2010. Una nueva especie de Cyclocephala Dejean de Perú, con la redescripción de C. spilopyga Erichson, 1847 y C. pygidiata Dupuis, 1999 (Coleoptera, Scarabaeidae, Dynastinae, Cyclocephalini). Entomotrópica, 25: 133-148.

Lachaume, G. 1992. Dynastinae Américains. Cyclocephalini - Agaocephalini - Pentodontini -Oryctini - Phileurini. Les Coleopteres du Monde 14. Sciences Nat; Venette, France. p. 1-56, 83-89, pl. 1-11.

Moore, M. R. 2011. Disentangling the phenotypic variation and pollination biology of the Cyclocephala sexpunctata species complex (Coleoptera: Scarabaeidae: Dynastinae). Thesis for the degree of Master of Science. Departament of Biological Sciences, Wichita State University, $180 \mathrm{p}$.

Morón, M. A. 1977a. Descripción del macho de Cyclocephala picta Burmeister, 1847 (Coleoptera: Melolonthidae: Dynastinae). Anales Instituto de Biología, Universidad Nacional Autónoma de México, Seria Zoología, 48: 133-140.

Morón, M. A. 1977b. Redescripción de Cyclocephala jalapensis Casey (Coleoptera: Melolonthidae: Dynastinae). Folia Entomológica Mexicana, 38: 19-28.

Ratcliffe, B. C. 2003. The Dynastinae scarab beetles of Costa Rica and Panamá. Bulletin of the University of Nebraska State Museum, 16: 1-506.

Ratcliffe, B. C. 2008. More new species of Cyclocephala from South America (Coleoptera: Scarabaeidae: Dynastinae). Coleopterists Bulletin, 62: 221-241.

Ratcliffe, B. C. \& Cave, R. 2006. The Dynastinae scarab beetles of Honduras, Nicaragua and El Salvador. Bulletin of the University of Nebraska State Museum, 21: 1-424.

Ratcliffe, B. \& Delgado-Castillo, L. 1990. New species and notes of Cyclocephala from Mexico (Coleoptera: Scarabaeidae: Dynastinae). Folia Entomológica Mexicana, 80: 41-57.

Restrepo, H., Morón, M. A., Vallejo, F., Pardo-Locarno, L. C. \& López-Ávila, A. 2003. Catálogo de Coleoptera Melolonthidae (Scarabaeidae-Pleurosticti) de Colombia. Folia Entomológica Mexicana, 42: $239-263$ 
True, J. R., Edwards, K. A., Yamamoto, D. \& Carroll, S. B. 1999. Drosophila wing melanin patterns form by vein-dependent elaboration of enzymatic prepatterns. Current Biology, 9: 1382-1391.

Young, R. M. \& Le Tirant, L. 2005. A new Cyclocephala from montane Colombia (Scarabaeidae: Dynastinae). Coleopterists Bulletin, 59:267-270.

Wittkopp, P. J., Carroll, S. B. \& Kopp, A. 2003. Evolution in black and white: genetic control of pigment patterns in Drosophila. Trends in Genetics, 19: 495-504. 